\title{
Bibliometric Analysis of the Solar Thermal System Control Methods
}

\author{
Mikelis DZIKEVICS $^{1 *}$, Dzintars JAUNZEMS ${ }^{2}$, Maris TERAUDS ${ }^{3}$ \\ ${ }^{1,2}$ Institute of Energy Systems and Environment, Riga Technical University, Azenes st. 12/1, Riga, LV-1048, \\ Latvia \\ ${ }^{3}$ Institute of Radioelectronics, Riga Technical University, Azenes st. 12, Riga, LV-1048, Latvia
}

\begin{abstract}
With the increase of renewable energy sources, the control methods of the energy systems are an increasingly important topic. Control methods have evolved from simple on/off controllers to methods that utilize artificial intelligence and can have a predictive nature. This paper investigates the control methods that are particular for solar thermal systems. A bibliometric method is applied to analyse scientific literature related to the specific application from the Web of Science database. Results show that recent developments in control methods focus on solar thermal to power and hybrid systems while district heating is a potential research gap. Annual publication trends, country, journal and keyword analysis is presented.
\end{abstract}

Keywords - District heating; literature gaps; model predictive control; smart controls

\begin{tabular}{|ll}
\hline Nomenclature & \\
MPC & Model predictive control \\
GS & Google Scholar \\
WoS & Web of Science \\
\hline
\end{tabular}

\section{INTRODUCTION}

Energy transition is one of the main steps towards climate neutrality covering energy efficiency and renewable energy topics. There is a need for comprehensive and mature innovations in all elements of energy systems from generation to end-user.

Solar thermal energy has become a widely applied technology and has been in development for decades. The main components of the solar energy collection have matured and further development in this field has to come from optimization, smarter control methods, hybrid applications, heat to power and power to heat principles or others. For the last years, there has been a trend of declining interest within the solar thermal market and continuous increase in the solar photovoltaic market and a drop in the price of respective technologies due to higher demand. Although the district heating sector is a large part of energy consumption in the world, some policies are looking at full electrification of this sector, thus leaving solar thermal technologies with less governmental aid. Nevertheless, some EU countries have created continuous growth opportunities in solar thermal installations mainly due to large installations for centralized or decentralized district heating [1].

* Corresponding author.

E-mail address: mikelis.dzikevics@rtu.lv

(C)2021 Mikelis Dzikevics, Dzintars Jaunzems, Maris Terauds.

This is an open access article licensed under the Creative Commons Attribution License (http://creativecommons.org/ licenses/by/4.0). 
Larger installations of solar thermal energy systems always are in combination with some other heating sources to cover the heating load throughout the year. Combining solar thermal energy with thermal storage and other heat sources provide new challenges, and often it is up to a district heating plant operator's experience and systems control algorithms to ensure continuous operation in a dynamic system. In many of these systems, traditional control methods are used, which provides an area of research to identify 'smarter' technologies or in other words, methods that are coming after the previous ones, that could assist in the operation of existing technologies and could help make these plants to be more flexible allowing to incorporate other emerging technologies.

In this study, the bibliometric analysis method is applied to identify control methods in the solar thermal field and developments of this field of research. The bibliometric method was considered mature in 2015 [2] and has become more widespread in the research fields where there is a sufficient number of publications. The initial aim of this method was to provide metrics for evaluating scientific production, but since then has been used by researchers for identifying fields that have not been sufficiently researched yet.

Bibliometric analysis has already been applied in studies that have analysed smart control applications in the thermal storage systems [3], [4] and solar cooling technologies [5] with the lateral concluding that it is highly recommended to combine this research method with extensive literature review. While the former presented the use of this method for identifying the most relevant journals for publishing in specific topics and use in identifying research gaps.

This study aims to analyse what control methods are being studied within all types of solar thermal applications, but with a specific focus on solar thermal collector use in district heating systems and applications of Model predictive control (MPC). Since this type of solar heating usage is limited compared to the overall usage of solar energy, this study is more focused on the control methods applied in other applications to look for possible research gaps, methods and uses that could be transferred over from other applications.

\section{METHODOLOGY}

This section describes the process of analysing existing literature in databases, the tools used, chosen keyword selection and other aspects that are analysed in this study. The main steps are illustrated in the following Fig. 1 and further described in more detail.

\subsection{Data Source}

The methodology of bibliometrics is based on analysing published literature in the scientific literature which is indexed in bibliographic databases. The main databases are Web of Science (WoS), Scopus, SciFinder and Google Scholar (GS). The two main databases that are used in the engineering field is WoS and Scopus [6].

Using datasets from multiple databases also add multiple challenges - how to correctly remove duplicates; how to make sure that the databases have entered all of the necessary information regarding the citation in their database; and how to analyse the citations. There are several tools available to carry out mentioned tasks, such as Bibliometrix, which is programmed in ' $R$ ' which is a language and environment for statistical computing and graphics [7]. ' $R$ ' can be upgraded with other R-packages [8]. This tool provides extensive possibilities in manipulating the collected data sets. However, previous studies have shown 
that Scopus and WoS databases overlap especially in engineering and computer science fields with $94 \%$ of WoS citations in Scopus [9]. The same study also concluded that while GS provides more sources than WoS and Scopus, but only around half of all the citations are from journals and also there is a lack of methods to reliably extract from GS. Therefore, the investigation in this article is based on an analysis of articles indexed in the WoS database.

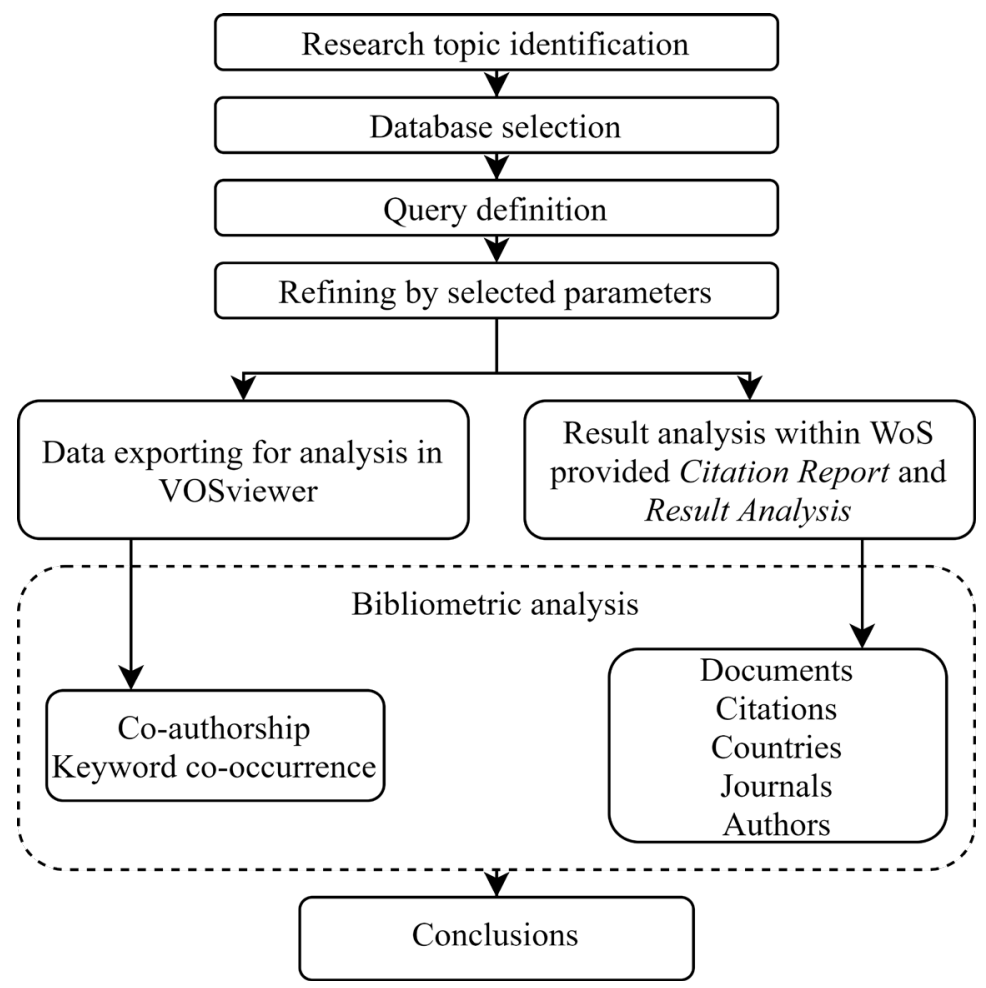

Fig. 1. Flowchart of the methodology.

\subsection{Tools}

Each database entry contains information that can be analysed - title, authors, journal, year, author's keywords, institution, abstract and used citations. This information can be used to apply mathematical methods to filter out articles that are not relevant to a specific case. It can be done based on the text each of the entries contains and in the result one can obtain a set of articles that relate to the specific question.

Specific tools have been developed to carry out not just by filtering, but also analysis of publication metrics, geographical attributes, author collaboration via co-authorship allowing to assess the main authors in the field, main research institutions and what collaboration exists within the field.

To carry out bibliometric analysis in this study a specialized computer program for bibliometric mapping VOSviewer [10] is used. VOS stands for visualization of similarities describing the intent of the tool. VOSviewer developed by Van Eck and Waltman has integrated the VOS mapping technique but also supports maps made from other statistical 
analysis tools such as SPSS. It is a freely available tool with open source code to promote the scientific nature for the public to have free access to evaluate the approaches used in this tool. To analyse data in VOSviewer from $\mathrm{WoS}$ in the manual it is suggested to use Tab-delimited file format.

WoS also provides citation analysis within the website to display data by WoS categories, publication years, document types, organizations, authors, countries and others. General information of the selected sources is provided which can be downloaded for further analysis.

\subsection{Data Collection}

This study analyses literature that is associated with control aspects of solar thermal systems. To obtain citations that are related to this topic a specific query can be used. Additionally, it is possible to refine by criteria such as publication years, WoS categories, document types, organizations, funding agencies, authors and title of the source.

The selected refined categories in this study are the period of 1995-2021 and document types - articles and proceedings. Document types such as books and review papers are excluded from this category to avoid recounting of citations. It should be noted that the selected time range can yield different results in publications and citations since new papers are published and old ones are cited more often. The latest papers also have less time to be found in the databases, thus resulting in a possibly lower article recognition and the number of citations. However, taking this into account in result analysis, authors see no reason to exclude the latest papers for this study (data acquisition is February-March 2021).

Two queries are initially used in the 'Topic Search', which looks for the words in the queries in the Title, Abstract, Keywords and Keywords Plus. The lateral is an algorithm that provides expanded terms stemming from references and the bibliography of the citation. The first query contains two phrases - 'Solar thermal' and 'control'. Quotation marks are used to look for phrases instead of individually selected words. In the second query number of specific control methods are named to look for citations that are more related to the discussion of controls. Search operators (AND, OR) are used to look for papers that include the phrase 'solar thermal', at least one of the specific control methods and the phrase 'control'.

To obtain a bibliography for the $3^{\text {rd }}$ query, an Advanced Search method within WoS is applied. This method allows looking for papers within a certain category field by using the $W C$ operator before the name of the category. In this query, citations are only from the category of Automation \& Control Systems. Topic (TS) operator looks for the phrase 'solar thermal' within the topic. The 'control' phrase is not used, since this category includes papers that are associated only with automation which implies control.

Lastly the $4^{\text {th }}$ query is used to identify specific use of model predictive control in the solar thermal systems and the $5^{\text {th }}$ query looks very specifically for solar thermal control applications in connection with district heating. Queries are summarized in the Table 1.

To obtain a bibliographic data file, an Export Record to File option is used. WoS allows exporting a maximum of 500 records at a time, therefore for the first query the data is split into two batches. WoS also allows choosing what content should be included - in this study Full Record and Cited References is used. These data files would then be directly used in VOSviewer.

The main information regarding the data source for the $1^{\text {st }}$ query is summarized in the Table 2 . 
TABLE 1. QUERY INFORMATION

\begin{tabular}{|c|c|c|c|}
\hline Nr. & Topic & Query in Web of Science & $\begin{array}{l}\text { Number } \\
\text { of } \\
\text { citations }\end{array}$ \\
\hline 1. & $\begin{array}{l}\text { Solar thermal and } \\
\text { control }\end{array}$ & 'Solar thermal' AND 'control' & 815 \\
\hline 2. & $\begin{array}{l}\text { Solar thermal and } \\
\text { mentioning of } \\
\text { specific control } \\
\text { methods }\end{array}$ & 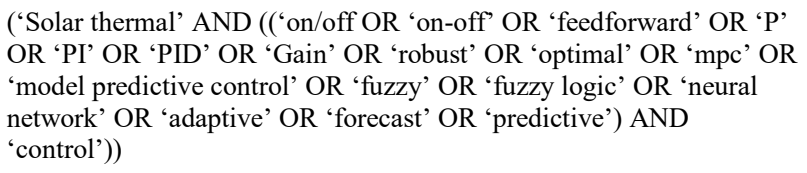 & 279 \\
\hline 3. & $\begin{array}{l}\text { Solar thermal in the } \\
\text { WoS category of } \\
\text { automation }\end{array}$ & $\mathrm{TS}=($ 'solar thermal') AND WC=Automation \& Control Systems & 88 \\
\hline 4. & $\begin{array}{l}\text { Model predictive } \\
\text { control application in } \\
\text { solar thermal } \\
\text { systems }\end{array}$ & (('Solar thermal' AND 'model predictive control')) & 41 \\
\hline 5. & $\begin{array}{l}\text { Control methods for } \\
\text { district heating }\end{array}$ & 'Solar thermal' AND 'control' AND 'district heating' & 32 \\
\hline
\end{tabular}

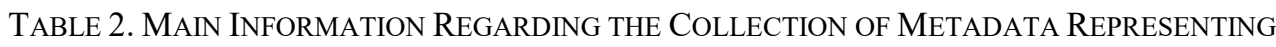
$1^{\text {ST }}$ QUERY

\begin{tabular}{ll}
\hline Description & Result \\
\hline Documents & 815 \\
Sources (Journals, Books, etc) & 379 \\
Time period & $1995-2021$ \\
Average years from publication & 6.01 \\
Keywords Plus & 1094 \\
Average citations per document & 12.66 \\
Authors & 2381 \\
Average Authors per Document & 2.92 \\
Average Co-Authors per Document & 3.74 \\
Documents Types & \\
Article & 467 \\
Proceedings paper & 311 \\
Article; proceedings paper & 26 \\
Article; book chapter & 6 \\
Article; early access & 5 \\
\hline
\end{tabular}

\subsection{Data Visualization}

Data visualization is carried out with the use of graphical representation of the direct data available from WoS and by use of VOSviewer.

In the case of data from WoS, there are two methods. For citations the following steps are taken:

1. The query is entered in WoS;

2. The data is refined for articles and proceedings; 
3. Use Create Citation Report in WoS;

4. If needed data is saved to Excel file for further graphical representation.

To acquire other types of information within WoS, steps 1 and 2 are repeated, but Result Analysis is selected as the next step. Here the rest of the analysis data are visualized in Treemap or Bar graph method and can be adjusted for how many results should be presented and minimum record count.

VoSviewer represents chosen and filtered data in three methods:

1. Network Visualization;

2. Overlay Visualization;

3. Density Visualization.

For each of these, there is a possibility to choose what weights are used based on what type of analysis is chosen. For example, if a co-occurrence map is being made, then weights are Links, Total Link Strength and Occurrences. Further, several visual adjustments can be used to better represent the data and prepare it so it can be shared within the articles.

\section{Results}

The query including citations that mention solar thermal and control phrases resulted in 815 citations (Table 1). More publications and citations within the topic start to appear around the year 2005. The number of publications peak is distinctive of the year 2018 (Fig. 2) with 111 and 53 for queries 1 and 2, respectively. A small drop in the number of annual publications was observed in the following years, indicating that the topic retains its relevance. There is a noticeable difference in the number of citations between the queries. The more specific $2^{\text {nd }}$ query sees a smaller, but stable number of citations, while the broader $1^{\text {st }}$ query has a noticeable drop in citations.

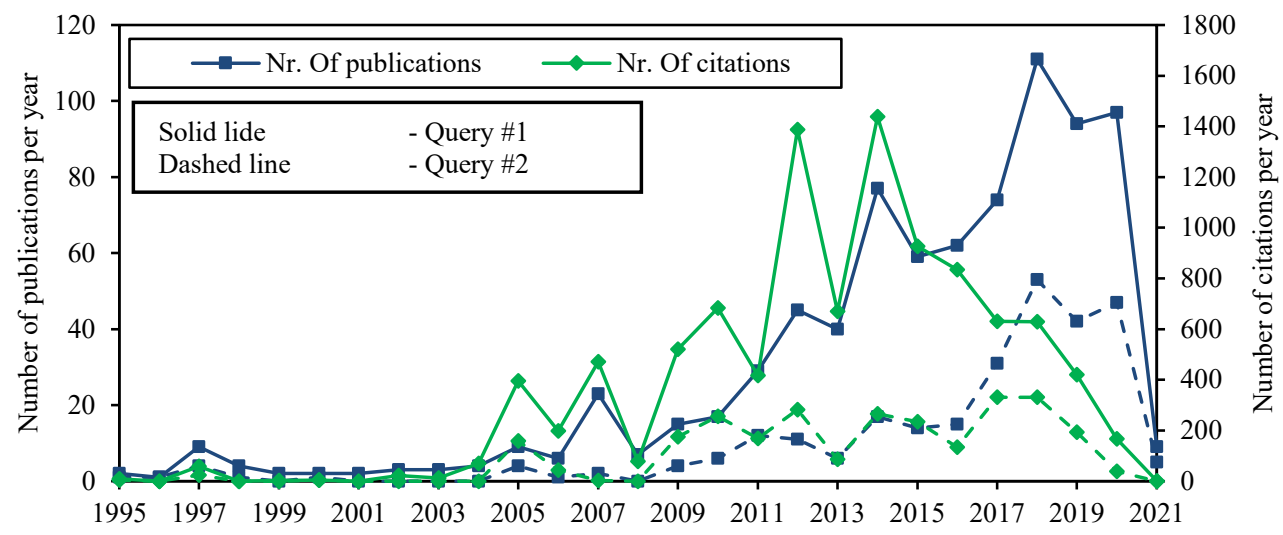

Fig. 2. The trend in the number of publications and citations with $1^{\text {st }}$ and $2^{\text {nd }}$ queries

Although USA, Germany and India are leading countries in the number of citations (Fig. 3), the latest publications are coming from Brazil, Belgium, Morocco, Chile and Turkey, indicating increased interest in the topic. According to categories given by WoS, 489 of the papers are under Energy and Fuels followed by the rest. The main journals under this category are Energy Procedia, Solar Energy, Renewable Energy, Applied Energy and others. 
Reviewing the most cited documents it can be observed, that many deals with the optimization of solar thermal systems in combination with building energy efficiency. 48 citations lay under the automation control systems category, indicating that only a fraction of papers has control as the main topic of research, and most deal with optimization and performance.

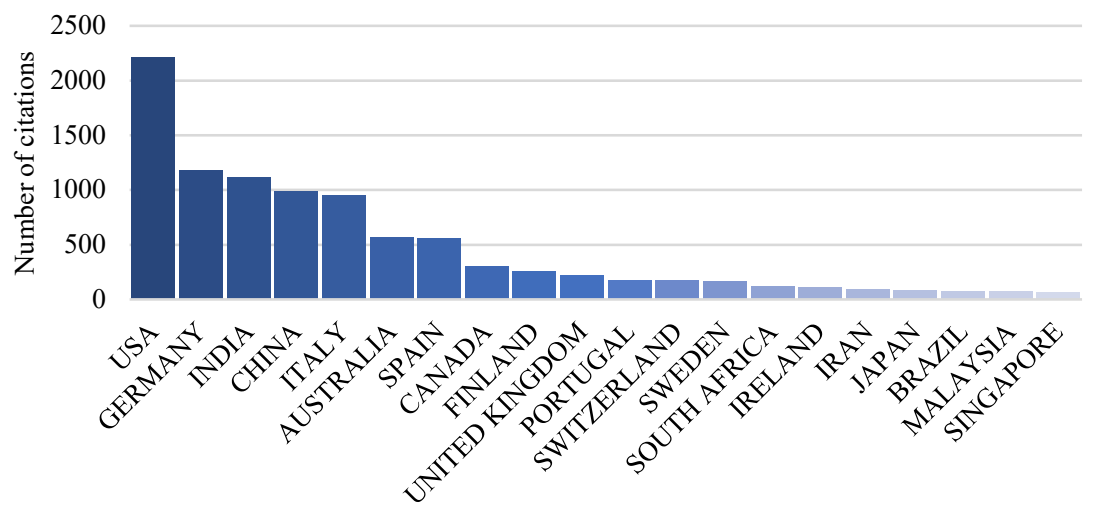

Fig. 3. Number of citations per country for $1^{\text {st }}$ query.

Looking at the co-authorship, only 13 authors meet the threshold of being an author of more than five papers within the $1^{\text {st }}$ query, 330 authors with two papers and 2634 authors with a single paper. The number of co-authorship is higher than the papers submitted because each paper has multiple authors. The trend of the falling number of citations agrees well with having few authors with a high number of citations in the year 2012, while there is a small number of authors with a lot of citations in the more recent years (Fig. 4(a)). Meanwhile, the number of documents (Fig. 4(b)) for authors shows a different distribution with a more even number of articles for each author with only a few standing out around the year 2018 .

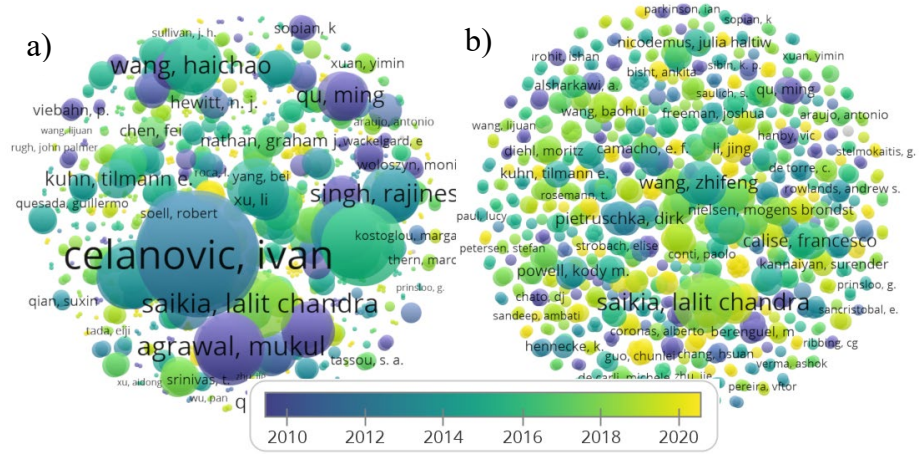

Fig. 4. Co-authorship representation for $1^{\text {st }}$ query for a) number of citations and b) number of documents representing the size of the circle and with average publication year representing the colour.

Keyword analysis of $1^{\text {st }}$ query found 3220 keywords, of which 174 meet the threshold of the minimum number of occurrences being above five. The three keywords with the most occurrences are performance (102 occurrences), design (85) and optimization (80). The keywords more directly connected with control with the highest occurrences are load 
frequency control (23), automatic-generation control (22) and predictive control (17). Citations with the control-related keywords are distinctively more recent with 2018 being the average year of publications where these keywords are most often used. The keywords map of $1^{\text {st }}$ query shows, that there are a lot of documents that do not directly look into control principles.

Applying $2^{\text {nd }}$ query results in 1475 keywords, of which 70 meet the threshold of five occurrences. The three most often used keywords are the same, but in a different order design (51), optimization (48) and performance (33). Since $2^{\text {nd }}$ query filtered out documents that are more closely related to control methods, the change is indicating that articles that focus on performance often look at other aspects than control, while the design is more closely related to developing control methods. The keyword load frequency control (21) is $5^{\text {th }}$ rank as the most popular occurrence, followed by automatic-generation control (21), predictive control (17), PID-controller (15) and model predictive control (14). There is a relatively smaller loss of occurrences in the above-mentioned keywords but a larger decrease in the number of articles submitted compared to $1^{\text {st }}$ query, indicating that filtering by specific control methods is a suitable approach for obtaining articles studying control methods.

Analysing further $2^{\text {nd }}$ query, it is possible to identify the main journals in this field. Fig. 5 illustrates that most documents largely correlate with the number of citations, but with some exceptions. Some journals such as the International Journal of Electrical Power \& Energy Systems have a relatively higher number of citations, compared to journal Energies, which have a high number of publications. Optics Express is another journal that has a high number of citations. Further analysis of the articles included in the $2^{\text {nd }}$ query that comes from this journal indicates that their topics are not closely related to the analysis of control methods. A high number of citations of these articles have introduced undesirable misrepresentation of data. For example, one of the papers applies to the spectral control, indicating that exclusion of term spectral would help to filter out such articles.

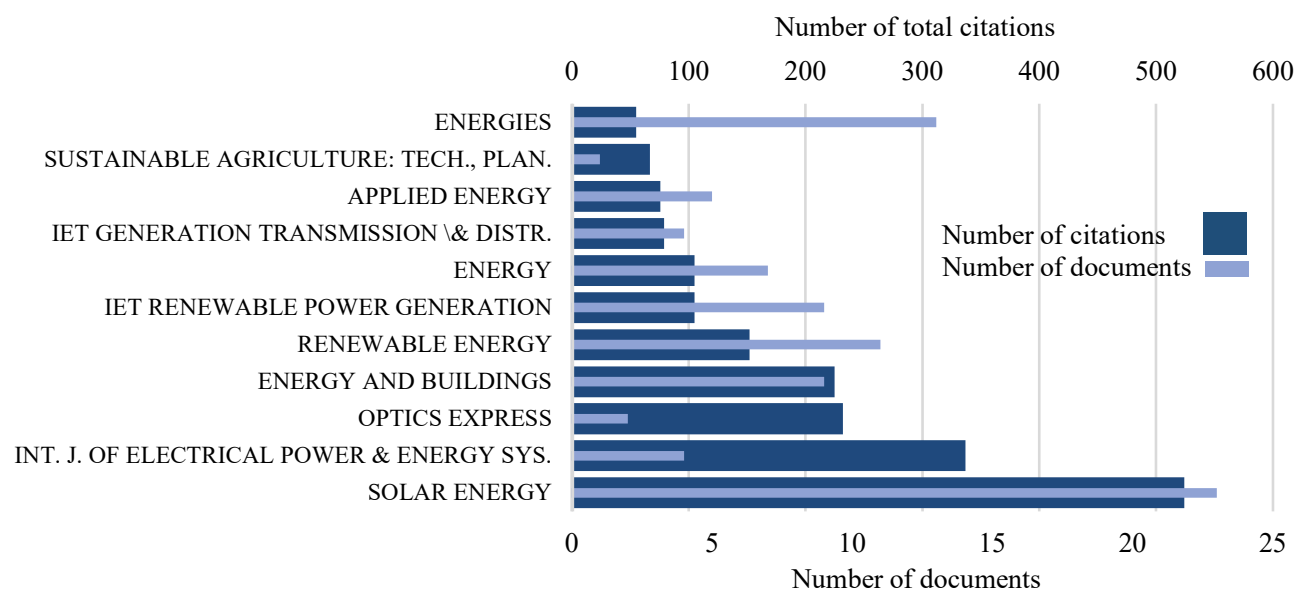

Fig. 5. Number of documents and citations for main journals for $2^{\text {nd }}$ query.

Analysis of $2^{\text {nd }}$ query's keywords co-occurrence with an increased threshold of 10 identifies 3 clusters (Fig. 6). The colours identify each cluster, while the distance between each keyword indicates how closely they are related. If the same keywords are more often used together, they will be closely related. The weight, in this case, is the occurrence, therefore the larger 
the size of the circle, the more often this keyword is used. Links between the keywords show which words are used together. Again, if they are used more often together, the lines get thicker. By applying this method, three clusters emerge - one where performance and optimization are closer related, another (blue in Fig. 6) related to control schemes and the third one which deals mostly with design and control methods. The keywords that are common to all clusters are the larger ones. These keywords do not help to identify specific categories, however looking at each cluster, some keywords with stronger links can be identified.

In the green cluster, two keywords describe the field of application - load frequency control, solar power stations and solar thermal power plants (hidden in Fig. 6 due to overlap). These systems are related to power generation and quality and indicates that the control methods within this cluster are mostly applied within this field. The most cited articles related to these keywords deal with solar thermal systems in combination with Stirling engines [11], [12], as part of hybrid systems [13], for load frequency control [14] and concentrated solar systems with molten-salt storage [15], [16] thus control methods within this cluster are applied to ensure stable electricity production.

The red cluster does not provide specific keywords indicating the field of application. However, by repeatedly applying only specific keywords within the $2^{\text {nd }}$ query it is possible to refine the list of articles. By refining with keywords storage, performance and optimization the most cited articles deal with hybrid systems [17] and performance analysis for systems for buildings and microgeneration [18] and desalination systems [19].

The blue cluster can be analysed by refining with the control schemes keyword, which results in 18 articles. The most cited articles deal with control schemes within parabolicthrough systems, hybrid and other systems, but are mainly concentrating on the control aspect, revealing that this keyword is important in finding articles related to control methods and modelling.

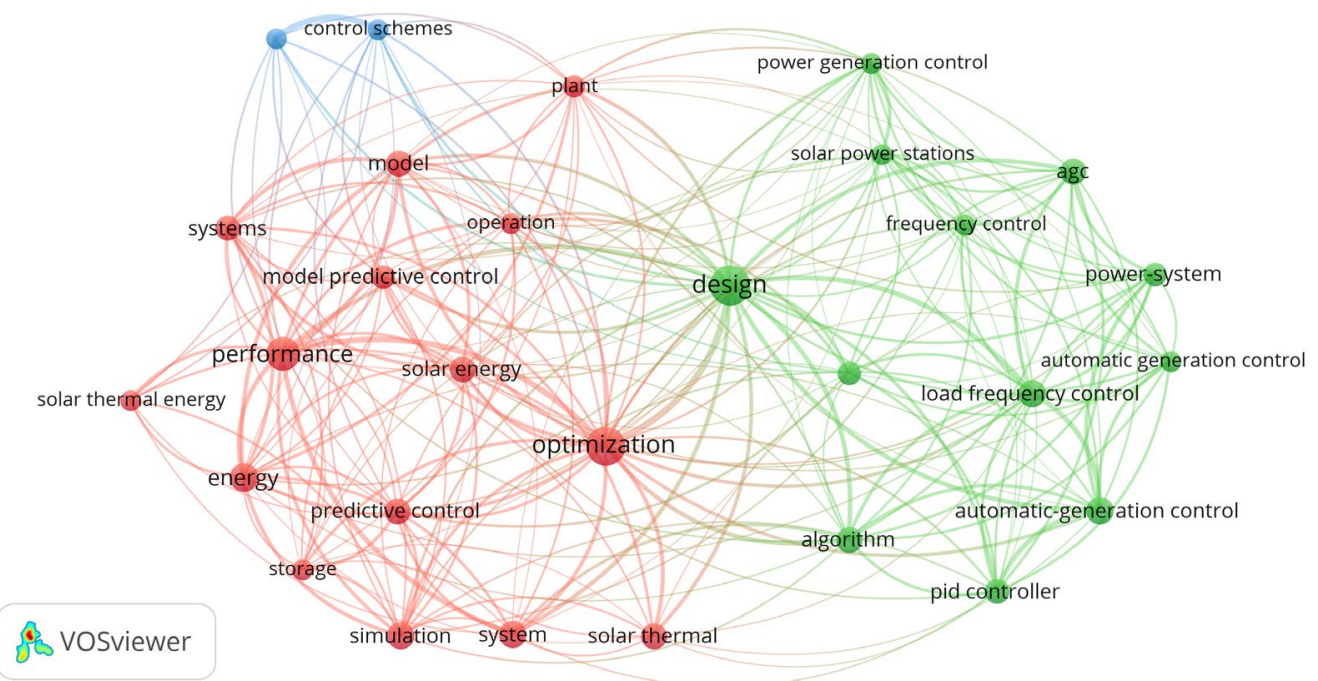

Fig. 6. Keyword co-occurrence network with the threshold of 10 or more occurrences with $2^{\text {nd }}$ query. 
The same three clusters can also be analysed regarding their average publication date (Fig. 7). Keywords such as design, optimization and performance are used for all types of topics, therefore their average date is the average of the represented period. However, less often used keywords provide more information. Keywords load frequency control, automaticgeneration control and PID control all have been used in recent studies, indicating that the use of classical controls such as PID are being applied in combination with more advanced control methods. Keywords solar thermal energy, storage, systems and solar thermal are the oldest ones indicating that lately fewer articles have been analysing control methods for these technologies.

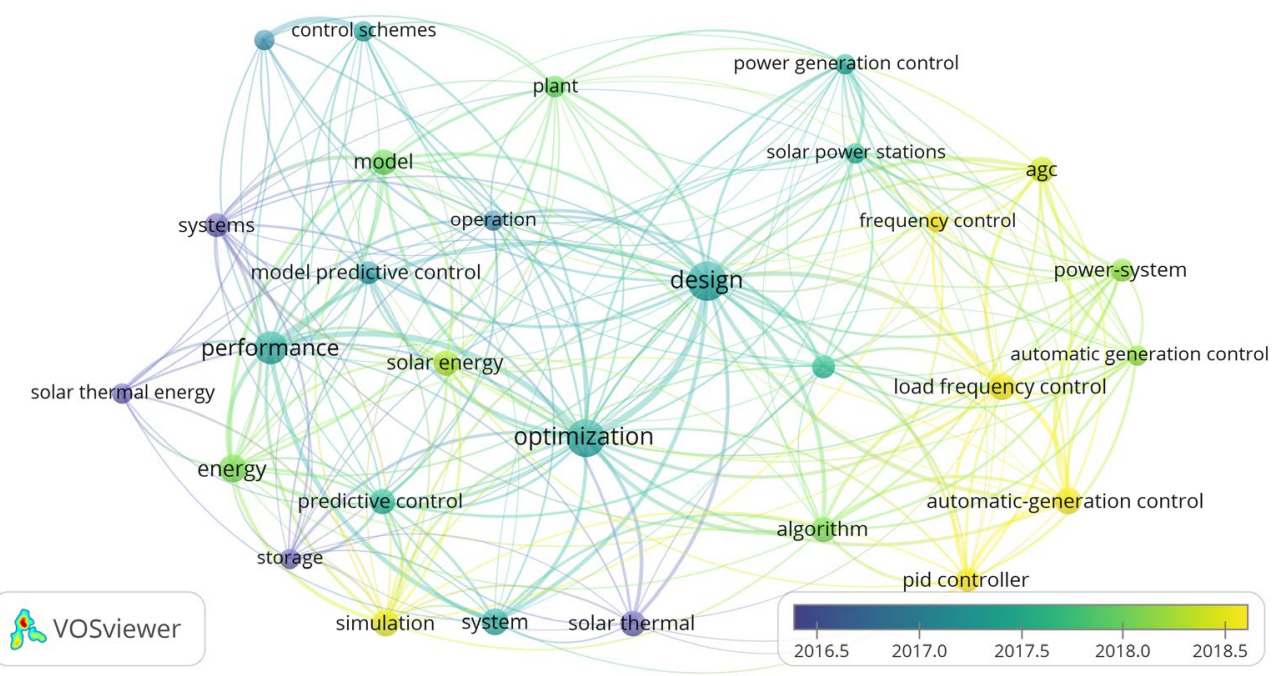

Fig. 7. Keyword co-occurrence average publication year with the threshold of 10 or more occurrences with $2^{\text {nd }}$ query.

Bibliographic coupling with sources as a unit of analysis is used to find journals with articles that represent the more relevant topics in the latest years. Filtering out occurrences below five provided a list of 24 journals. The journals with the most recent publications are journals that are under the WoS category of Automation \& Control Systems. To analyse control methods within energetic systems, $3^{\text {rd }}$ query restricts articles from this specific category within WoS.

From the 353 keywords within articles from applying the $3^{\text {rd }}$ query, the main keywords with the highest occurrences are optimization (8), solar energy (8) and design (6). The control methods with the highest occurrences are predictive control (5) and model predictive control (4) which also occur in more recent studies (Fig. 8). MPC use in design and optimization shows up in recent studies, however, MPC use in the control of aspects closer connected with collector fields is a possible research gap. Most cited articles deal with modelling and control of dynamic systems for a variety of solar thermal systems, but mainly solar thermal power plants. As expected, this indicates that journals that are specifically related to automation and control can be better content based, however at the same time they can be a more limited source of articles. 


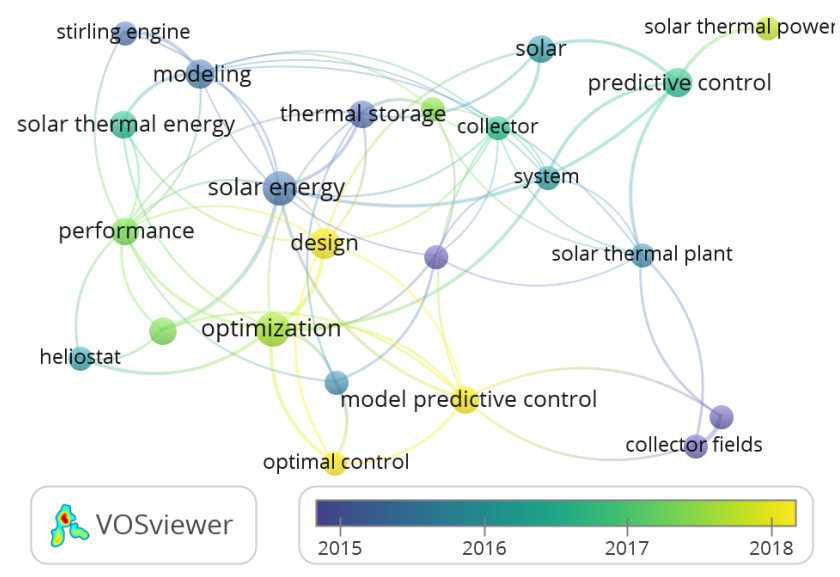

Fig. 8. Keyword co-occurrence average publication year with the threshold of 2 or more occurrences with 3rd query.

To analyse the further application of model predictive control in the solar thermal systems the $4^{\text {th }}$ query limits the citations to 41 articles that deal specifically with this control method. The network between the main keywords (Fig. 9) can be related to the topics of the most cited articles. The topics that lead in citations are the use of MPC in solar assisted heat pumps (blue cluster), gasification (purple cluster), control of collectors (green cluster), thermal storage and buildings (red cluster), overheating protection for collectors (cyan cluster). Related to most of the articles are keywords design, simulation and energy (yellow cluster) as represented by their placement close to the middle. Results indicate that the use of the MPC method is being studied for a variety of applications.

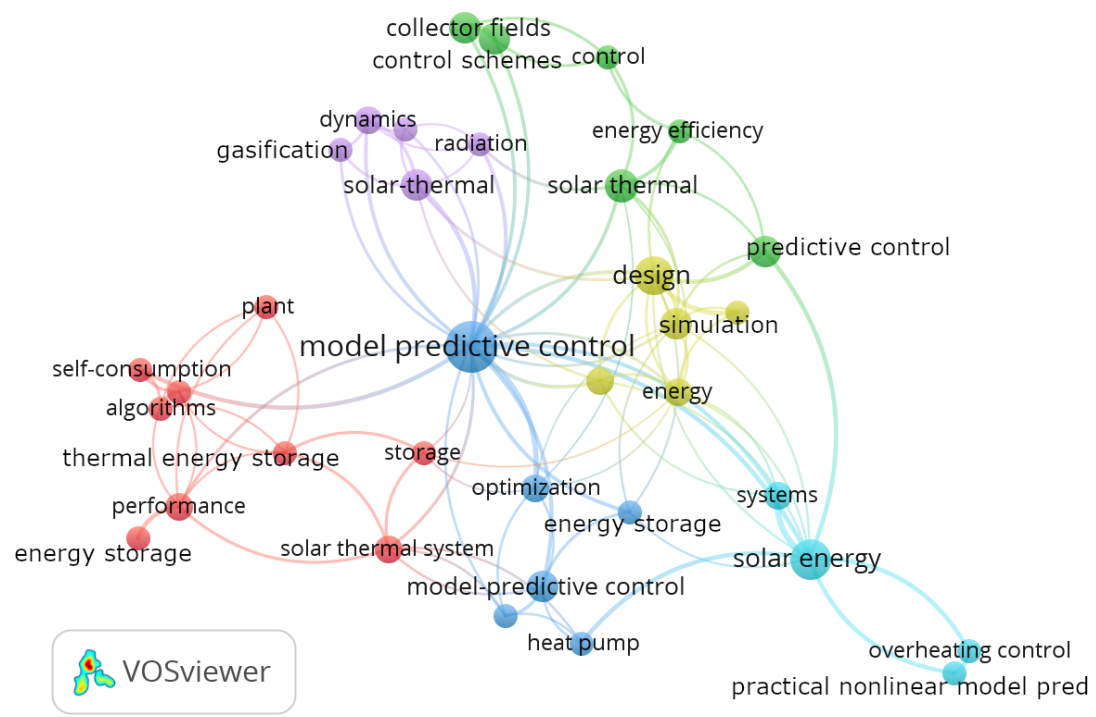

Fig. 9. Keyword co-occurrence network with the threshold of 2 or more occurrences with $4^{\text {th }}$ query.

The final $5^{\text {th }}$ query is specifically looking at solar thermal systems used for district heating and results in 32 articles with a total of 401 citations. This query also peaked in the year 2018 , 
but the number of published documents is still above values before 2018 indicating that the topic is still growing. Energy Procedia holds most of the papers (10), followed by the $16^{\text {th }}$ International Symposium on District heating and cooling DHC2018 (4) explaining peak in 2018, followed by Applied Energy, Energies and Renewable Energy (3 each). The leading countries are Germany, Finland, Sweden and Austria.

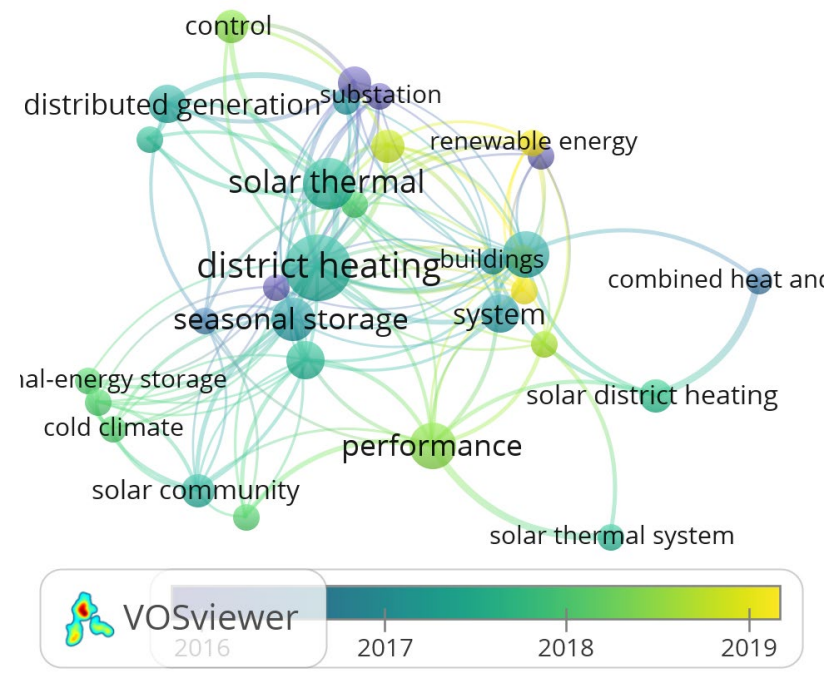

Fig. 10. Keyword co-occurrence average publication year with the threshold of 2 or more occurrences with $5^{\text {th }}$ query.

There are 189 keywords used, but only 31 meet the threshold of a minimum of two occurrences (Fig. 10). Looking at the keywords with mentioned occurrences, none relate to the specific control method. Looking at all the keywords, the control appears in multiple locations - logic control for introducing thermal storage in the system; control strategies for long term storages and increase in performance; control analysis within mathematical models; control schemes for parabolic through systems; switching control for heat flows and control validation for experimental systems.

\section{Conclusions}

An often-used approach with the traditional literature analysis method is combined with the inclusion of more keywords to obtain results with a very limited number of articles that are closely related to the keywords used. However, this approach excludes the rest of the articles that could provide useful information into control methods being studied in related fields. An alternative approach would be to analyse a query with less specific keywords, however, it can be time-consuming if the query results in a high number of articles.

The bibliometric analysis is a useful tool to visualize the main topics being studied. Network view indicates what control methods and what are the aims of the studies in different applications. Overlay view indicates what topics are either no longer studied or have not been fully explored. In this study, 5 queries were used with consequent queries being more restrictive than the previous ones.

The $1^{\text {st }}$ query provides information on the state of studies that are in some way connected to solar thermal systems and control methods and results in 815 articles. Analysis of source countries indicates that together with a few leading countries (USA, Germany, India), there 
is a developing scene of new studies coming from less represented countries (Brazil, Belgium, Morocco, Chile and Turkey). $2^{\text {nd }}$ query limits the articles that deal with specific control methods mentioned in the query and results in 279 articles. Analysis of trends for both queries indicates that the control aspect itself has become more popular starting from the year 2005 and is still a largely developing topic with increasing readers interest. This topic in recent years is being studied more by the new researchers which is indicated by the share of only around $12 \%$ and $15 \%$ of authors with more than one article for queries 1 and 2, respectively.

Analysis of the journals and specific articles within the $2^{\text {nd }}$ query illustrates the use of additional filtering of results and possibilities for improvements of queries. All of the used queries don't use any exclusions, while analysis shows that exclusion of terms such as spectral control would remove articles that have a weaker connection with control methods of readers interest.

Analysis of keywords indicate that two methods applied in this study are favourable in emitting articles with a weak link to the control methods - use of a specific control method names in the query ( $2^{\text {nd }}$ query) or searching within more specific journals or categories $\left(3^{\text {rd }}\right.$ query) if such are available. However, the lateral approach can greatly reduce available literature and result in the loss of important information. Keywords networks and analysis of most cited articles within these networks indicate that specific control methods are more analysed for power generation and quality applications, while for performance and optimization only predictive controls stand out as keywords.

Model predictive control (MPC) is more studied within the category of Automation \& Control Systems used in the $3^{\text {rd }}$ query and shows up in most recent articles. However, it does not show strong links with particular applications except control of collector fields, which has not been studied in recent years, providing a possible research gap. $4^{\text {th }}$ query did not limit category but was limited to articles that studied the usage of MPC. The resulted network indicated wide applications of MPC for the design and optimization of systems such as heat pumps, gasification, control of collectors, thermal storage and buildings and overheating protection for collectors.

The $5^{\text {th }}$ query removes limitations of specific control methods and looks at applications of any control methods in the combination of district heating. Keyword control shows up in multiple areas but without strong indications of specific control methods, rather than the usage of control in general. This however indicates that there is a research gap and control methods such as MPC could be further studied for solar thermal use in district heating.

\section{ACKNOWLEDGEMENT}

This research is funded by the Latvian Council of Science, project 'Smart control applications in thermal energy storage systems (TEScon)', project No. 1zp-2020/2-0113.

\section{REFERENCES}

[1] Weiss W., Spörk-Dür M. Solar Heat Worldwide 2020 - Global market development and trends in 2019. Sci. Technol. Built Environ. 2019:24(8):819.

[2] Ellegaard O., Wallin J. A. The bibliometric analysis of scholarly production: How great is the impact? Scientometrics 2015:105(3):1809-1831. https://doi.org/10.1007/s11192-015-1645-Z

[3] Tarragona J., de Gracia A., Cabeza L. F. Bibliometric analysis of smart control applications in thermal energy storage systems. A model predictive control approach. J. Energy Storage 2020:32:101704. https://doi.org/10.1016/j.est.2020.101704

[4] Calderón A., et al. Where is Thermal Energy Storage (TES) research going?-A bibliometric analysis. Sol. Energy 2019:200:37-50. https://doi.org/10.1016/j.solener.2019.01.050

[5] Saikia K., et al. A bibliometric analysis of trends in solar cooling technology. Sol. Energy 2020:199:100-114. https://doi.org/10.1016/j.solener.2020.02.013 
[6] Li J., et al. Citation analysis: Comparison of web of science ${ }^{\circledR}$, scopusTM, scifinder ${ }^{\circledR}$, and google scholar. J. Electron. Resour. Med. Libr. 2010:7(3):196-217. https://doi.org/10.1080/15424065.2010.505518

[7] Aria M., Cuccurullo C. Bibliometrix: An R-tool for comprehensive science mapping analysis. J. Informetr. 2017:11(4):959-975. https://doi.org/10.1016/j.joi.2017.08.007

[8] CoreTeam R. R: A Language and Environment for Statistical Computing. Vienna: R Foundation for Statistical Computing, 2017:2.

[9] Martín-Martín A., et al. Google Scholar, Web of Science, and Scopus: A systematic comparison of citations in 252 subject categories. 2018:12(4):1160-1177. https://doi.org/10.1016/j.joi.2018.09.002

[10] Van Eck N. J., Waltman L. Software survey: VOSviewer, a computer program for bibliometric mapping. Scientometrics 2010:84:523-538. https://doi.org/10.1007/s11192-009-0146-3

[11] Rahman A., Saikia L. C., Sinha N. AGC of dish-Stirling solar thermal integrated thermal system with biogeography based optimised three degree of freedom PID controller. IET Renew. Power Gener. 2016:10(8):1161-1170. https://doi.org/10.1049/iet-rpg.2015.0474

[12] Tasnin W., Saikia L. C. Maiden application of an sine-cosine algorithm optimised FO cascade controller in automatic generation control of multi-area thermal system incorporating dish-Stirling solar and geothermal power plants. IET Ren. Pow. Gen. 2018:12(5):585-597. https://doi.org/10.1049/iet-rpg.2017.0063

[13] Barik A. K., Das D. C. Proficient load-frequency regulation of demand response supported bio-renewable cogeneration based hybrid microgrids with quasi-oppositional selfish-herd optimisation. IET Gener. Transm. Distrib. 2019:13(13):2889-2898. https://doi.org/10.1049/iet-gtd.2019.0166

[14] Saha A., Saikia L. C. Utilisation of ultra-capacitor in load frequency control under restructured STPP-thermal power systems using WOA optimised PIDN-FOPD controller. IET Gener. Transm. Distrib. 2017:11(13):3318-3331. https://doi.org/10.1049/iet-gtd.2017.0083

[15] Cirocco L. R., et al. Controlling stored energy in a concentrating solar thermal power plant to maximise revenue. IET Ren. Pow. Gener. 2015:9(4):379-388. https://doi.org/10.1049/iet-rpg.2014.0141

[16] Menéndez R. P., et al. A novel modeling of molten-salt heat storage systems in thermal solar power plants. Energies 2014:7(10):6721-6740. https://doi.org/10.3390/en7106721

[17] Das D. C., Roy A. K., Sinha N. GA based frequency controller for solar thermal-diesel-wind hybrid energy generation/energy storage system. Int. J. Electr. Power Energy Syst. 2012:43(1):262-279. https://doi.org/10.1016/j.ijepes.2012.05.025

[18] Dorer V., Weber R., Weber A. Performance assessment of fuel cell micro-cogeneration systems for residential buildings. Energy Build. 2005:37(11):1132-1146. https://doi.org/10.1016/j.enbuild.2005.06.016

[19] Chang H., et al. Modeling and optimization of a solar driven membrane distillation desalination system. Renew. En. 2010:35(12):2714-2722. https://doi.org/10.1016/j.renene.2010.04.020 